\title{
The Discernment of Ekiti Ethnic Tribe of South Western Nigeria on Wild Edible Vegetables
}

\author{
Joshua Kayode ${ }^{1}$, Modupe Janet Ayeni ${ }^{1}$, Eunice Damilola Akinbinu ${ }^{1}$, Grace Damilola \\ Ogunrotimi $^{1}$ \\ ${ }^{I}$ Department of Plant Science and Biotechnology,Ekiti State University, Ado-Ekiti, Nigeria \\ joshua.kayode@eksu.edu.ng
}

\begin{abstract}
The discernment of Ekiti people on the wild edible vegetables (WEVs) was conducted in the three senatorial districts of Ekiti State through interviews using a semistructured questionnaire matrix. The interviews were focused, conversational and two-way in communication. Also the abundance of each of the identified WEVs was determined. The respondents transcend sex, age and literacy status though most of them were females, mostly adults of over 25 years, illiterates, mostly of low economic status and engaged in agricultural activities yet these socio-economic classifications were not pre-requisites to their consciousness on the WEVs. A total of 51 WEVs belonging to 30 families were valued for medicine and nutrition in the study area with the family Asteraceae having the highest number of species. The leaves constituted the major part used in the identified WEVs. The diseases managed and/prevented by these WEVs were classified into 37 groups while 40 of the WEVs were recognized as sources of vitamins and minerals by the respondents. The need to ensure sustainability of the WEVs in a manner that would improve the dietary and health conditions of the indigenous people of the study area was identified.
\end{abstract}

Keywords: discernment; Ekiti Ethnic Tribe; wild edible vegetables; sustainability

\section{Introduction}

Wild edible vegetables (WEVs) are vegetables that grow spontaneously in selfmaintaining populations in natural or semi-natural ecosystems (Campton, 2008). They exist independently of direct human actions. Nnamani et al. (2017) opined that some of these vegetables do not require formal cultivation as they easily grow in the wild and are readily available in the field. According to Modi et al. (2006), WEVs contribute significantly to the dietary requirements of rural households. Though information about their contributions to world nutrition is still limited (Kassim, 2009) yet Kalemba, (2007) opined that the promotion of utilization and commercialization of indigenous WEVs could provide a viable alternative to sustainable livelihood and food security.

The WEVs are nutritious (Vaishali et al. 2013), delicious and cheap. They are also valued as traditional medicine (Adebooye and Opabode, 2004; Saqib et al., 2011) and used for prevention of most prevalent life style diseases (KDAH 2015). WEVs now constitute an integral part of the culture of indigenous people of Africa. The indigenous knowledge on them is passed on from generation to generations (Lwoga et al. 2010).

Recent initiative revealed that the consumption of WEVs is declining, particularly in Nigeria (Ogunrotimi et al. 2018). Some people still regard them as weeds and vegetables of low status (Bvenura and Afolayan 2019). All these served as disincentives to their cultivation. Consequent on these, the need for proper documentation of the wild edible vegetables among the various ethnic groups in the country and the discernment of these indigenous people on them cannot be over-emphasized, particularly now that massive and diverse anthropogenic factors are prevalent and eroding the vegetation of the country. Thus a gradual loss of genetic diversity persists on WEVs and this may deprive the future generations of these useful resources (Ogunrotimi et al. 2018). 
emails: birex.journal@gmail.com

The Ekiti is an indigenous Yoruba tribe in Ekiti State, in the south western part of Nigeria. This study being reported here aimed to identify the discernment of Ekiti people on the WEVs in their environment.

\subsection{The Study Area}

\section{Materials and Methods}

Ekiti State is situated entirely in the south western part of Nigeria, between Long. $5^{\circ} 00^{1}$ and $6^{\circ} 00^{1}$ East and Lat. $7^{\circ} 25^{1}$ and $8^{\circ} 20^{1}$ North of the equator. The State which has a total land area of about $7000 \mathrm{~km} 2$ (Kayode 1999, Obembe and Kayode 2019), enjoys a tropical climate with two distinct seasons, the dry seasons, from November to March, and the raining season from April to October (Kayode et al., 2016). Temperature ranged between $21^{\circ} \mathrm{C}$ and $38^{\circ} \mathrm{C}$ (Arowosegbe et al., 2018). The State has a population of 2,384,212 (NPC, 2010). Agriculture is the major occupation of Ekiti people which provide income and employment for more than $75 \%$ of the population (Arowosegbe et al., 2018, Adedokun and Kayode 2019).

\subsection{Methods}

\section{a. Ethno-botanical Survey and Collection of Data:}

This study was conducted in the three senatorial districts of Ekiti State, namely; Ekiti Central, Ekiti North and Ekiti South. Two Local Government areas (LGAs) were selected from each senatorial district and four rural areas were purposively selected from each LGA, making a total of six LGAs and twenty-four rural communities. 5 respondents, each of whom has maintained domicile in the community for a continuous period of 10 years were purposively selected in each community and interviewed using a semi-structured questionnaire matrix. The interviews were focused, conversational and two-way in communication.

Information regarding the local names of WEVs in the study area, parts used and their cultivation status was documented. Also respondents' perceived medicinal and nutritional values were documented.

\section{b. Determination of the Abundance of the identified WEVs}

After the completion of the inventory stated above, the abundance of each of identified species in each LGA was determined by random selection of two of the already selected communities in the LGA. In each of the selected community a 4-point scale was used to define the abundance of each of the identified WEVs species. This was awarded using the time taken to physically encounter the species in each community (Bonger and Popma 1988).

Identified WEVs found in less than an hour was regarded as 'Abundant' and awarded 3 Points, those found between 1 to $2 \mathrm{hrs}$ was regarded as 'Frequent' and awarded 2 Points, those found between $2 \mathrm{hrs}$ and $5 \mathrm{hrs}$ was regarded as 'Occasional' and awarded 1 Point. Identified plant not found after 5 hrs was regarded as 'Rare' and awarded 0 Point

The average of the total points obtained on each of the identified plant species was regarded as the species abundance status.

\section{Results}

Table 1 revealed that the respondents transcend sex, age and literacy status though most of them were females (65\%), mostly adults of over 25 years and illiterates $(60 \%)$. Though the index of wealth used in this study varied from one community to another yet most of the respondents were of low economic status and were mostly engaged in agricultural activities.

The respondents were quite conscious of the WEVs in their environment and their medicinal and nutritional values. Thus, the socio-economic classifications of the respondents were not regarded as pre-requisites to their consciousness on the WEVs in their environment. 
Budapest International Research in Exact Sciences (BirEx) Journal Volume 2, No 2, April 2020, Page: 125-135 e-ISSN: 2655-7827 (Online), p-ISSN: 2655-7835(Print) www.bircu-journal.com/index.php/birex emails: birex.journal@gmail.com

Table 1. Socio-economic classification of respondents sampled in Ekiti State, Nigeria

\begin{tabular}{|l|l|l|}
\hline Feature & Description & $\begin{array}{l}\text { Proportion (\%) of } \\
\text { Respondents }\end{array}$ \\
\hline \multirow{2}{*}{ Sex } & Male & 35 \\
\cline { 2 - 3 } & Female & 65 \\
\hline \multirow{2}{*}{ Age } & $25-50$ & 40 \\
\cline { 2 - 3 } & $>50 y$ yrs & 60 \\
\hline \multirow{2}{*}{ Literacy } & Literate & 40 \\
\cline { 2 - 3 } & Illiterate & 60 \\
\hline Status & Resource Rich & 35 \\
\hline \multirow{2}{*}{ Occupation } & Resource Poor & 65 \\
\cline { 2 - 3 } & Agricultural & 60 \\
\cline { 2 - 3 } & Non - & 40 \\
& Agricultural & \\
\hline
\end{tabular}

A total of $51 \mathrm{WEVs}$ belonging to 30 families were observed to be valued for medicine and nutrition in the study area (Table 2) while Asteraceae has the highest number of species (9), 4 each were members of the families Amarantaceae, Euphorbiaceae and Solanaceae. 3 were members of the family of Portulacaceae, 2 each were members of the family of Malvaceae and Papillionaceae. Other families possessed a species each. 31 (61\%) species of the identified species were herbs, $12(24 \%)$ were shrubs and $8(16 \%)$ were trees. The leaves constituted the major part used. The results obtained revealed that a total of 27 (53\%) species have their leaves used as vegetables, another 17 (33\%), $1(3 \%)$ and $1(3 \%)$ have their leaves and stems, leaves and fruits, leaves and tubers respectively, used as vegetables. Other parts used as vegetables were fruits and tubers. $6(12 \%)$ of the identified wild edible vegetables were cultivated species, 20 (39\%) were uncultivated but grow as wild while 25(49\%) were capable of being cultivated but also grow as wild.

Table 3 shows the medicinal value of the wild edible vegetable species. The diseases managed and/ prevented by these identified wild vegetables were classified into 37 groups. Table 4 shows the nutritional values of the wild edible vegetables sampled in Ekiti State, most of the vegetables were valued as sources of vitamins and minerals. 40 of the identified

Table 2. Checklist of Vegetables Identified in the rural Areas of Ekiti State, Nigeria

\begin{tabular}{|c|c|c|c|c|c|c|}
\hline $\mathrm{S} / \mathrm{n}$ & Botanical Names & $\begin{array}{c}\text { Local/Vernacular } \\
\text { Names }\end{array}$ & Family & $\begin{array}{l}\text { Growth } \\
\text { Form }\end{array}$ & Part Used & Cultivation \\
\hline 1 & Abelmoschus esculentus & Ila, Ilasa, Ewe-ila & Malvaceae & Herb & Leaves & $\begin{array}{l}\text { Cultivated \& } \\
\text { Uncultivated }\end{array}$ \\
\hline 2 & Acacia seyal & Aluki, Sie, Siyi & Mimosaceae & Tree & Leaves & Uncultivated \\
\hline 3 & $\begin{array}{l}\text { Acanthospermum } \\
\text { hispidum }\end{array}$ & $\begin{array}{l}\text { Dangunro, } \\
\text { Gbadagiri, Egun- } \\
\text { igba }\end{array}$ & Asteraceae & Shrub & $\begin{array}{l}\text { Leaves and } \\
\text { Stem }\end{array}$ & Uncultivated \\
\hline 4 & Adansonia digitata & Oshe, Ose & Bombacaceae & Tree & Leaves & Uncultivated \\
\hline 5 & Aframomum melegueta & $\begin{array}{l}\text { Oburo, Ata-ire, } \\
\text { Itaye }\end{array}$ & Zingiberacea & Herb & Rhizome & $\begin{array}{l}\text { Cultivated \& } \\
\text { Uncultivated }\end{array}$ \\
\hline 6 & Amaranthus dubius & $\begin{array}{l}\text { Tete abalaye/ } \\
\text { Atetedaye }\end{array}$ & Amarantaceae & Herb & $\begin{array}{l}\text { Leaves and } \\
\text { Stem }\end{array}$ & $\begin{array}{l}\text { Cultivated \& } \\
\text { Uncultivated }\end{array}$ \\
\hline 7 & Amaranthus spinosus & Dagunro & Amaranthaceae & Herb & $\begin{array}{l}\text { Leaves and } \\
\text { Stem }\end{array}$ & Uncultivated \\
\hline 8 & Annona senegalensis & $\begin{array}{l}\text { Abo, Afe, Epo, } \\
\text { Arere, Ibobo }\end{array}$ & Annonaceae & Shrub & Leaves & Uncultivated \\
\hline
\end{tabular}


Budapest International Research in Exact Sciences (BirEx) Journal

Volume 2, No 2, April 2020, Page: 125-135

e-ISSN: 2655-7827 (Online), p-ISSN: 2655-7835(Print)

www.bircu-journal.com/index.php/birex

\begin{tabular}{|c|c|c|c|c|c|c|}
\hline \multicolumn{7}{|c|}{ emails:birex.journal@gmail.com } \\
\hline 9 & Argemone Mexicana & $\begin{array}{l}\text { Ahun-ekun, Ekan- } \\
\text { ekun, Egun-arugbo }\end{array}$ & Papaveraceae & Herb & $\begin{array}{l}\text { Leaves and } \\
\text { Stem }\end{array}$ & Uncultivated \\
\hline 10 & Basella alba & $\begin{array}{l}\text { Amunututu, Popo } \\
\text { safara, Gbagana }\end{array}$ & Basellaceae & Herb & Leaves & $\begin{array}{l}\text { Cultivated \& } \\
\text { Uncultivated }\end{array}$ \\
\hline 11 & Bidens pilosa & $\begin{array}{l}\text { Abere oloko, Omo } \\
\text { langanran, } \\
\text { Agomonyan, Ewe } \\
\text { abere }\end{array}$ & Asteraceae & Herb & Leaves & $\begin{array}{l}\text { Cultivated \& } \\
\text { Uncultivated }\end{array}$ \\
\hline 12 & Bridelia ferruginea & $\begin{array}{l}\text { Ira, Iya, Asagbo, } \\
\text { Araasa, Ida-Odan }\end{array}$ & Euphorbiaceae & Shrub & $\begin{array}{l}\text { Leaves and } \\
\text { Stem }\end{array}$ & Uncultivated \\
\hline 13 & Cajanus cajan & $\begin{array}{l}\text { Feregede, Otili, } \\
\text { Sese were }\end{array}$ & Papilionaceae & Shrub & $\begin{array}{l}\text { Leaves and } \\
\text { fruits }\end{array}$ & Uncultivated \\
\hline 14 & Calotropis procera & Bomubomu & Apocynaceae & Shrub & Leaves & $\begin{array}{l}\text { Cultivated \& } \\
\text { Uncultivated }\end{array}$ \\
\hline 15 & Ceiba pnetandra & $\begin{array}{l}\text { Araba, Eegun, } \\
\text { Eegungun, } \\
\text { Ponpola, Odere }\end{array}$ & Malvaceae & Tree & Leaves & Uncultivated \\
\hline 16 & Celosia argentea & $\begin{array}{l}\text { Shoko, } \\
\text { Shokoyokoto }\end{array}$ & Amaranthaceae & Herb & $\begin{array}{l}\text { Leaves and } \\
\text { Stem }\end{array}$ & $\begin{array}{l}\text { Cultivated \& } \\
\text { Uncultivated }\end{array}$ \\
\hline 17 & Celosia trigyna & $\begin{array}{l}\text { Sepososun, } \\
\text { Ajefowo, } \\
\text { Ajemawofo }\end{array}$ & Amaranthaceae & Herb & $\begin{array}{l}\text { Leaves and } \\
\text { Stem }\end{array}$ & $\begin{array}{l}\text { Cultivated \& } \\
\text { Uncultivated }\end{array}$ \\
\hline 18 & $\begin{array}{l}\text { Cnidoscolus } \\
\text { aconitifolius }\end{array}$ & Iyana ipaja & Euphorbiaceae & Shrub & Leaves & $\begin{array}{l}\text { Cultivated \& } \\
\text { Uncultivated }\end{array}$ \\
\hline 19 & Colocasia esculenta & $\begin{array}{l}\text { Koko, Ewe-koko, } \\
\text { Koko funfun }\end{array}$ & Araceae & Shrub & Leaves & \\
\hline 20 & Corchorus olitorus & $\begin{array}{l}\text { Ewedu, Ooyo, } \\
\text { Eeyo }\end{array}$ & Tiliaceae & Herb & Leaves & $\begin{array}{l}\text { Cultivated \& } \\
\text { Uncultivated }\end{array}$ \\
\hline 21 & $\begin{array}{l}\text { Crassocephalum } \\
\text { crepidioides }\end{array}$ & Ebolo, Ebire & Asteraceae & Herb & Leaves & $\begin{array}{l}\text { Cultivated \& } \\
\text { Uncultivated }\end{array}$ \\
\hline 22 & Cucurbita maxima & $\begin{array}{l}\text { Apala, Elegede, } \\
\text { Esun,Isin }\end{array}$ & Cucurbitaceae & Herb & $\begin{array}{l}\text { Leaves and } \\
\text { Fruits }\end{array}$ & $\begin{array}{l}\text { Cultivated \& } \\
\text { Uncultivated }\end{array}$ \\
\hline 23 & Erigeron floribundus & $\begin{array}{l}\text { Olowonjeja, } \\
\text { Arowojenja }\end{array}$ & Asteraceae & Shrub & Leaves & $\begin{array}{l}\text { Cultivated \& } \\
\text { Uncultivated }\end{array}$ \\
\hline 24 & Hibiscus sabdariffa & Isapa, Shapa & Malvaceae & Herb & Fruits & $\begin{array}{l}\text { Cultivated \& } \\
\text { Uncultivated }\end{array}$ \\
\hline 25 & Ipomoea batatas & Odunkun, Anamo & $\begin{array}{l}\text { Convolvulacea } \\
\text { e }\end{array}$ & Herb & Tuber & $\begin{array}{l}\text { Cultivated \& } \\
\text { Uncultivated }\end{array}$ \\
\hline 26 & Jatropha curcas & $\begin{array}{l}\text { Ewe lapalapa, } \\
\text { Botuje, Isofe, } \\
\text { Polopolo }\end{array}$ & Euphorbiaceae & Herb & Leaves & $\begin{array}{l}\text { Cultivated \& } \\
\text { Uncultivated }\end{array}$ \\
\hline 27 & Lactuca taraxacifolia & $\begin{array}{l}\text { Yanrin, Efo } \\
\text { gbenuoke }\end{array}$ & Asteraceae & Herb & Leaves & Uncultivated \\
\hline 28 & Lagenaria siceraria & $\begin{array}{l}\text { Igba, Ugba, Itakun } \\
\text { agba }\end{array}$ & Curspitaceae & Herb & Leaves & Uncultivated \\
\hline 29 & Launaea taraxacifolia & $\begin{array}{l}\text { Yanrin, Yamurin, } \\
\text { Itakin-igba }\end{array}$ & Asteraceae & Herb & $\begin{array}{l}\text { Leaves and } \\
\text { Stem }\end{array}$ & $\begin{array}{l}\text { Cultivated \& } \\
\text { Uncultivated }\end{array}$ \\
\hline 30 & Leptadenia hastate & $\begin{array}{l}\text { Iran-aji, Isanaje- } \\
\text { igbo }\end{array}$ & Apocynaceae & Herb & Leaves & Uncultivated \\
\hline 31 & Manihot esculenta & Ege, Gbaguda & Euphorbiaceae & Shrub & Leaves and & Cultivated \& \\
\hline
\end{tabular}


Budapest International Research in Exact Sciences (BirEx) Journal

Volume 2, No 2, April 2020, Page: 125-135

e-ISSN: 2655-7827 (Online), p-ISSN: 2655-7835(Print)

www.bircu-journal.com/index.php/birex

emails: birex.journal@gmail.com

\begin{tabular}{|c|c|c|c|c|c|c|}
\hline & & & & & $\begin{array}{l}\text { ex.journal@gme } \\
\text { Tuber }\end{array}$ & $\frac{\mathrm{om}}{\text { Uncultivated }}$ \\
\hline 32 & Myrianthus arboreus & $\begin{array}{l}\text { Ibeshere, Ebiseghe, } \\
\text { Ewe-ade }\end{array}$ & Urticaceae & Tree & Leaves & Uncultivated \\
\hline 33 & Ocimum gratissimum & Efinrin & Lamiaceae & Shrub & Leaves & $\begin{array}{l}\text { Cultivated \& } \\
\text { Uncultivated }\end{array}$ \\
\hline 34 & Phaseolus lunatus & $\begin{array}{l}\text { Awuje, Ere, } \\
\text { Papala, Popondo }\end{array}$ & Papilionaceae & Herb & Leaves & $\begin{array}{l}\text { Cultivated \& } \\
\text { Uncultivated }\end{array}$ \\
\hline 35 & Physalis angulata & $\begin{array}{l}\text { Koropo, Elefopo, } \\
\text { Eleti, Efopo }\end{array}$ & Solanaceae & Herb & Leaves & Uncultivated \\
\hline 36 & Piper guineense & Iyere, Uziza & Piperaceae & Tree & \begin{tabular}{|l|} 
Leaves and \\
Fruits \\
\end{tabular} & Uncultivated \\
\hline 37 & Portulaca oleracea & $\begin{array}{l}\text { Papasan, Esinsan- } \\
\text { omode, Senolapo }\end{array}$ & Portulacaceae & Herb & Leaves & Uncultivated \\
\hline 38 & Senecio biafrae & Worowo & Asteraceae & Climber & \begin{tabular}{|l|} 
Leaves and \\
Stem
\end{tabular} & $\begin{array}{l}\text { Cultivated \& } \\
\text { Uncultivated }\end{array}$ \\
\hline 39 & Sesamum radiatum & $\begin{array}{l}\text { Ekuku-gogoro, } \\
\text { Eku, Aparun }\end{array}$ & Pedaliaceae & Herb & \begin{tabular}{|l} 
Leaves and \\
Stem \\
\end{tabular} & Uncultivated \\
\hline 40 & Solanecio biafrae & $\begin{array}{l}\text { Gbologi, Bologi, } \\
\text { Worowo }\end{array}$ & Asteraceae & Herb & Leaves & Uncultivated \\
\hline 41 & Solanum americanum & Odu & Solanaceae & Herb & \begin{tabular}{|l} 
Leaves and \\
Stem
\end{tabular} & $\begin{array}{l}\text { Cultivated \& } \\
\text { Uncultivated }\end{array}$ \\
\hline 42 & Solanum macrocarpon & $\begin{array}{l}\text { Okerekere, } \\
\text { Igbagba, Gbagba }\end{array}$ & Solanaceae & Herb & $\begin{array}{l}\text { Leaves and } \\
\text { Stem }\end{array}$ & $\begin{array}{l}\text { Cultivated \& } \\
\text { Uncultivated }\end{array}$ \\
\hline 43 & Solanum nigrum & Efo-odu & Solanaceae & Herb & $\begin{array}{l}\text { Leaves and } \\
\text { Stem }\end{array}$ & $\begin{array}{l}\text { Cultivated \& } \\
\text { Uncultivated }\end{array}$ \\
\hline 44 & Solanum scabrum & Ogunmo, Ogunma & Solanaceae & Herb & $\begin{array}{l}\text { Leaves and } \\
\text { Stem }\end{array}$ & $\begin{array}{l}\text { Cultivated \& } \\
\text { Uncultivated }\end{array}$ \\
\hline 45 & Spondias mombin & $\begin{array}{l}\text { Akika, Okikan, } \\
\text { Iyeye, Olosan }\end{array}$ & Anacardiaceae & Tree & Leaves & Uncultivated \\
\hline 46 & Talinum triangulare & Gbure & Portulacaceae & Herb & $\begin{array}{l}\text { Leaves and } \\
\text { Stem }\end{array}$ & $\begin{array}{l}\text { Cultivated \& } \\
\text { Uncultivated }\end{array}$ \\
\hline 47 & Telfairia occidentalis & $\begin{array}{l}\text { Ugu, Ugwu, } \\
\text { Apiroko }\end{array}$ & Portulacaceae & Herb & \begin{tabular}{|l} 
Leaves and \\
Stem \\
\end{tabular} & $\begin{array}{l}\text { Cultivated \& } \\
\text { Uncultivated }\end{array}$ \\
\hline 48 & $\begin{array}{l}\text { Thaumatococcus } \\
\text { daniellii }\end{array}$ & $\begin{array}{l}\text { Ewe eran, Ewe } \\
\text { iran, Ewe Ojuku }\end{array}$ & Marantaceae & Shrub & Leaves & Uncultivated \\
\hline 49 & $\begin{array}{l}\text { Triplochiton } \\
\text { scleroxylon }\end{array}$ & Arere, Eruku, Aifo & Sterculiaceae & Tree & Leaves & Uncultivated \\
\hline 50 & Vernonia amygdalina & Ewuro & Asteraceae & Shrub & Leaves & $\begin{array}{l}\text { Cultivated \& } \\
\text { Uncultivated }\end{array}$ \\
\hline 51 & Vitex doniana & Oori, Oori nla & Verbenaceae & Tree & \begin{tabular}{|l} 
Leaves and \\
Fruts
\end{tabular} & $\begin{array}{l}\text { Cultivated \& } \\
\text { Uncultivated }\end{array}$ \\
\hline
\end{tabular}

Table 3. Medicinal values of the vegetables sampled in Ekiti State, Nigeria

\begin{tabular}{|c|l|l|}
\hline S/n & \multicolumn{1}{|c|}{ Medicinal values } & \multicolumn{1}{c|}{ Vegetable species } \\
\hline 1 & $\begin{array}{l}\text { Manages malaria/ } \\
\text { fever }\end{array}$ & $\begin{array}{l}\text { A.dubius, C.olitorius, J.curcas, L. taraxacifolia, P. lunatus, } \\
\text { P.angulata, V.doniana, V. amygdalina }\end{array}$ \\
\hline 2 & $\begin{array}{l}\text { Lowers blood } \\
\text { pressure/ treat } \\
\text { hypertension }\end{array}$ & $\begin{array}{l}\text { B.alba, M. esculenta, P.oleracea, S. biafrae, S. macrocarpon, S. } \\
\text { nigrum, V. amygdalina }\end{array}$ \\
\hline 3 & Boost blood & A. hispidum, B. alba, C. esculenta, C. cajan, C. maxima, C. \\
\hline
\end{tabular}


Budapest International Research in Exact Sciences (BirEx) Journal

Volume 2, No 2, April 2020, Page: 125-135

e-ISSN: 2655-7827 (Online), p-ISSN: 2655-7835(Print)

www.bircu-journal.com/index.php/birex emails: birex.journal@gmail.com

\begin{tabular}{|c|c|c|}
\hline & & $\begin{array}{l}\text { olitorius, } P . \text { lunatus, } P . \text { angulata, } P . \text { oleraceae, S. biafrae, } S . \\
\text { americanum, S. nigrum, T. triangulare }\end{array}$ \\
\hline 4 & $\begin{array}{l}\text { Safe delivery/ Ease } \\
\text { Delivery }\end{array}$ & C. cajan, C. olitorius, $M$. arboreus, S. radiatum, T.triangulare \\
\hline 5 & \begin{tabular}{|l|} 
Cures gastro- \\
intestinal disorder
\end{tabular} & $\begin{array}{l}\text { A.seyal, B. alba, C.cajan, C. pentandra, C. trigyna, C. olitorius, } \\
\text { C. maxima, L. hastata, M. arboreus, P.angulata }\end{array}$ \\
\hline 6 & Cures pile & C.olitorius, S, scabrum \\
\hline 7 & Infertility problem & A.mexicana, V. amygdalina \\
\hline 8 & Respiratory problem & A.digitata, V. doniana, V. amygdalina \\
\hline 9 & Skin infection & $\begin{array}{l}\text { A.mexicana, B.alba, C. aconitifolius, C. pentandra, C. trigyna, } \\
\text { J. curcas, L. taraxacifolia, L. siceraria, M. esculenta, S. } \\
\text { americanum }\end{array}$ \\
\hline 10 & $\begin{array}{l}\text { Cures rheumatism } \\
\text { and arthritis }\end{array}$ & A.seyal, A.hispidum, A.melegueta, J. curcas \\
\hline 11 & Cures epilepsy & A.senegalensis, S.biafrae \\
\hline 12 & Cures toothache & J. curcas, L. taraxacifolia \\
\hline 13 & Cures leprosy & A. seyal, A. mexicana, L. hastate \\
\hline 14 & Treat cold & A.seyal, A.melegueta, A.senegalensis, C.pentandra, L.hastata \\
\hline 15 & Treatment of cancer & $\begin{array}{l}\text { A. hispidum, B.pilosa, } C \text {. olitorius, } M . \text { esculenta, } M . \text { arboreus, } \\
\text { P. oleracea, S. scabrum, V. doniana }\end{array}$ \\
\hline 16 & Antidote to poison & L. sciceraria \\
\hline 17 & Treatment of asthma & P. angulata \\
\hline 18 & Cures heamorhages & A.seyal, A.dubius, C.cajan, \\
\hline 19 & $\begin{array}{l}\text { Liver, bladder and } \\
\text { kidney disease }\end{array}$ & $\begin{array}{l}\text { A. digitata, A. dubius, A. mexicana, B. pilosa, C. trigyna, } C \text {. } \\
\text { trigyna, }\end{array}$ \\
\hline 20 & Use as condiment & A.melegueta \\
\hline 21 & $\begin{array}{l}\text { Menstrual pain } \\
\text { treatment }\end{array}$ & A.melegueta \\
\hline 22 & Convulsion treatment & A.senegalensis \\
\hline 23 & $\begin{array}{l}\text { Sexual transmitted } \\
\text { disease/ venereal } \\
\text { disease treatment }\end{array}$ & $\begin{array}{l}\text { A. senegalensis, C.pentandra, C.aconitifolius, C.olitorius, } \\
\text { L.hastata }\end{array}$ \\
\hline 24 & $\begin{array}{l}\text { Stomach ache } \\
\text { treatment }\end{array}$ & $\begin{array}{l}\text { A.senegalensis, C. cajan, C. trigyna, M. esculenta, P.angulata, } \\
\text { P. oleracea, S. obtusifolia, S.nigrum }\end{array}$ \\
\hline 26 & Contraceptive & C. pentandra \\
\hline 27 & $\begin{array}{l}\text { Heart complaint, } \\
\text { troubles/ heart pain } \\
\text { treatment }\end{array}$ & C. trigyna, S. americanum, S.macrocarpon \\
\hline 28 & $\begin{array}{l}\text { Treatment of } \\
\text { scorpion sting/ insect } \\
\text { bite }\end{array}$ & C. aconitifolius, S.radiatum \\
\hline 29 & Insomnia treatment & C. aconitifolius \\
\hline 30 & Treat alcoholism & C. aconitifolius \\
\hline 31 & Treaty diabetes & C. aconitifolius, I. batatas, L. taraxacifolia \\
\hline 32 & $\begin{array}{l}\text { Sore eyes and Ear } \\
\text { aches treatment }\end{array}$ & S. obtusifolia, S. macrocarpon, $V$. doniana \\
\hline 33 & Ulcers treatment & C.maxima, S. nigrum \\
\hline 34 & Measles treatment & I.batatas, L. taraxacifolia, T. tiangulare, V. amygdalina \\
\hline 35 & $\begin{array}{l}\text { Treatment of } \\
\text { constipation }\end{array}$ & L.taraxacifolia, S. biafrae, S.macrocarpon \\
\hline
\end{tabular}


Budapest International Research in Exact Sciences (BirEx) Journal

Volume 2, No 2, April 2020, Page: 125-135

e-ISSN: 2655-7827 (Online), p-ISSN: 2655-7835(Print)

www.bircu-journal.com/index.php/birex emails: birex.journal@gmail.com

\begin{tabular}{|c|l|l|}
\hline 36 & $\begin{array}{l}\text { Aids bowel } \\
\text { movement/ Aids } \\
\text { digestion }\end{array}$ & A.dubius, C. trigyna, T. triangulare \\
\hline 37 & $\begin{array}{l}\text { Fight diseases/ } \\
\text { protect the body }\end{array}$ & $\begin{array}{l}\text { B. ferruginea, C.olitorius, S.radiatum, T. triangulare, } V . \\
\text { amygdalina }\end{array}$ \\
\hline
\end{tabular}

Table 4. Nutritional values of the vegetables sampled in Ekiti State, Nigeria

\begin{tabular}{|c|c|c|}
\hline $\mathrm{S} / \mathrm{n}$ & $\begin{array}{l}\text { Nutritional value/ } \\
\text { Nutritive value }\end{array}$ & Vegetable species \\
\hline 1. & $\begin{array}{l}\text { Source of vitamin } \\
\text { and mineral }\end{array}$ & $\begin{array}{l}\text { seyal, A. digitata, A. melegueta, A. dubius, B. pilosa. C. cajan, } C . \\
\text { procera, C. petandra, C. trigyna, C. aconitifolius, C. esculenta, C. } \\
\text { olitorius, C. maxima, E. floribundus, I. batatas, J. curcas, L. } \\
\text { taraxacifolia, L. siceraria, L. hastata, M. esculenta, M. arboreus, } \\
\text { P. lunatus, } P . \text { angulata, } P \text {. oleraceae, S. radiatum, S. biafrae, S. } \\
\text { macrocarpon, S. nigrum, S. scrabum, S. mombin, T. triangulare, T. } \\
\text { scleroxylon, V. amygdalina, V. doniana }\end{array}$ \\
\hline 2 & For strong bone & $\begin{array}{l}\text { pilosa, C. cajan, C. trigyna, C. aconitifolius, C. maxima, I. batatas, } \\
\text { L. taraxacifolia, S. obtusifolia, S.macrocarpon, C. micranthum, C. } \\
\text { acontifolius, S.americanum, S. nigrum, S. macrocarpon }\end{array}$ \\
\hline 3 & $\begin{array}{l}\text { Rich in dietary } \\
\text { fiber }\end{array}$ & $\begin{array}{l}\text { pilosa, } C . \text { cajan, } C . \text { trigyna, } C . \text { acontifolius, } C . \text { maxima, I. batatas, } \\
\text { taraxacifolia, } S . \text { americanum, } S . \text { nigrum, } S . \text { macrocarpon, } S . \\
\text { monbin, } T . \text { triangulare, } V . \text { doniana }\end{array}$ \\
\hline 4 & $\begin{array}{l}\text { Strength/ Energy } \\
\text { to the body }\end{array}$ & $\begin{array}{l}\text { seyal, } A . \text { digitata, A. melegueta, A. dubius, B. pilosa, C. cajan, } C . \\
\text { trigyna, } C . \text { aconitifolius, } C . \text { maxima, I. batatas, L. taraxacifolia, } S . \\
\text { americanum, S. nigrum, S. macrocarpon, S. monbin, } T . \\
\text { triangulare, V. doniana, V.amygdalina }\end{array}$ \\
\hline 5 & Source of protein & $\begin{array}{l}\text { ligitata, A. dubius, B. pilosa, C.cajan, C. trigyna, J. curcas, L. } \\
\text { taraxacifolia, L. siceraria, L. hastata, P. lunatus, P. angulata, } \\
\text { T.triangulare }\end{array}$ \\
\hline 6 & For smooth skin & mexicana, C. trigyna, C. aconitifolius, C. olitorius, J. curcas \\
\hline 7 & $\begin{array}{l}\text { For cleaning of } \\
\text { body system }\end{array}$ & $\begin{array}{l}\text { hispidum, A. senegalensis, } C \text {. micranthum, C. olitorius, } P . \text { angulata, } \\
\text { P. lunatus, P. olerscea, S. biafrae, S. americanum, V. amygdalina }\end{array}$ \\
\hline
\end{tabular}

Table 5. Abundance status of the identified WEV species in Ekiti State, Nigeria

\begin{tabular}{|c|c|}
\hline $\begin{array}{l}\text { Abundance } \\
\text { Status }\end{array}$ & Identified Wild Edible Vegetable Species \\
\hline Abundant & 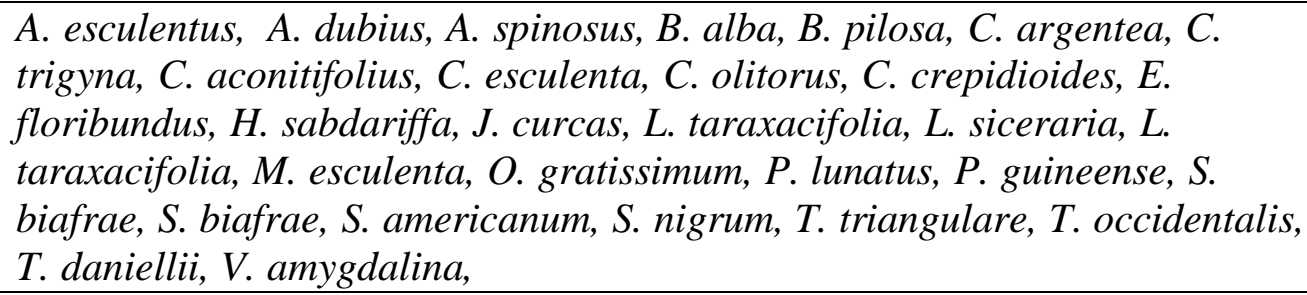 \\
\hline Frequent & $\begin{array}{l}\text { C. cajan, } C \text {. procera, } C \text {. maxima, I. batatas, L. hastata, P. angulata, } S . \\
\text { radiatum, S. macrocarpon, S. scabrum, S. mombin }\end{array}$ \\
\hline Occasional & A. seyal, A. hispidum, A. melegueta, A. mexicana, B. ferruginea, P. oleracea \\
\hline Rare & $\begin{array}{l}\text { A. digitata, A. senegalensis, } T \text {. scleroxylon, V. doniana, C. pentandra, } M \text {. } \\
\text { arboreus. }\end{array}$ \\
\hline
\end{tabular}

were recognized as sources of vitamins and minerals by the respondents. 14 vegetables were valued for strengthening of bones, 21 for strengthening the body, 16 for dietary fibers, 21 for protein, 5 for enhancing smoothness of skin and 11 for cleansing of the stomach system.

DOI: https://doi.org/10.33258/birex.v2i2.869 
$57 \%$ of the identified WEVs were abundant, $20 \%$ were frequent and $12 \%$ each were occasional and rare on the abundance scale used in this study (Table 5). While the abundant species were mostly herbs, most of which germinated as weeds, the rare species were mostly tree species.

\section{Discussion}

This study revealed that the Ekiti ethnic tribe of south west in Nigeria, like other tribes in the country, still valued WEVs in their environment. Most of the WEVs are freely available within the natural habitats of Ekiti State. Table 1 revealed that the respondents transcend socioeconomic classifications. This observation supports the previous assertions of Kayode et al., (2015) and Ayeni et. al. (2018) that socio-economic factors were not pre-requisites to the consciousness of the indigenous people on the economic and ecological values of plants in their vicinities.

51 wild edible vegetables species belonging to 30 families were documented (Table 2). Thus a wide range of species diversity abounds on WEVs among the Ekiti indigenous tribe. Elsewhere in Nigeria, wide diversity of edible vegetables was reported (Abubaker et. al. 2012, Ajiboye et. al. 2014, Ogunrotimi et. al.2018). The family Asteraceae had the largest number of species. Most of these species were herbaceous and grow as wilds. Kayode (2005) asserted that the ecological success of the Asteraceae could be attributed to their methods of reproduction. Many of them were annual plants and reproduced by seeds. The seeds are usually dispersed by air. Even when these seeds were dispersed during unfavorable conditions, they then exhibit dormancy that is easily broken during favourable conditions (Oberbaner and Strain 1985). The ability to exhibit dormancy was described as a survival strategy in the members of the family Asteraceae (Harper 1977). Recent initiative revealed that Asteraceae now constituted the largest plant family (Quora 2019). Ojelel and Kakudidi (2015) also revealed that these plants possessed higher tolerance to weather changes hence they are available for most part of the year. The leaves constituted the major parts widely utilized in the identified WEVs for vegetables thus supporting the previous observation made in most previous studies undertaken in Nigeria, such as the studies of Ali et al. (2008) and Kebede et al. (2017).

The perception of the indigenous Ekiti tribe revealed that many of the identified WEVs were of medicinal values. Field observation revealed that many of them were deliberately eaten to manage or prevent the incidences of diseases supposedly cure or prevented by these vegetables. These diseases were classified into over 30 types in this study. Field observation however revealed that in the consumption of these vegetables, emphases were placed on prevention rather than cure. Also the field observation revealed that a gross dearth of orthodox medicine abounds in most of the communities used in this study and where they are available, their costs were exorbitant and far beyond the reach of the rural dwellers. Thus dwellers saw the wild vegetables as nature's way of enhancing health management. This observation tends to concur with the opinion of Coolborn et al. (2018) that Ekiti tribe relied on the use of herbs and believed on their effectiveness with no mild effect on the human body system.

Similarly the perceptions of the respondents on the nutritional importance of the identified WEVs were equally revealed in this study (Table 4). The vegetables were believed to be sources of minerals, vitamins, protein and energy. They were equally valued for their ability to maintain teeth, bones and skin. Field observation revealed that the Ekiti diets are mainly of staple foods. Labadarios et al. (2005) observed that these foods lacked dietary diversity, which results in nutrient deficiencies. Thus, according to Modi et al.(2006), Chach and Oluoch (2002), the WEVS offered the potential to improve nutrients intake when consumed. Van der Walt et al. (2005) asserted that the vegetables require simple technologies and input to grow and cultivated. Other factors that might serve as incentives to their utilities include their nutrient contents, accessibility, affordability and acceptance by the indigenous people of the study area (Mauder and Meaker, 2007).

Most of the identified WEVs in this study were abundant in status (Table 5), the 
abundance was skewed to the herbaceous vegetables. Tree species -A. digitata, A. senegalensis, T. scleroxylon, V. doniana, C. pentandra and M. arboreus-were observed to be rare in the study area. Thus with the increasing anthropogenic factors in the study area, the need for conservation of these species cannot be over-emphasized.

\section{Conclusion}

Some of the identified species will require domestication. Cultivation of some of the presently uncultivated species should be embarked upon. Deforestation must be controlled (Neudeck et al. 2012). At present in the south western Nigeria, a gross dearth of labour hands proliferates thus accelerating the use of fire for vegetation clearance especially when preparing new farms. This must be checked. These will ensure sustainability of the WEVs in a manner that would improve the dietary and health conditions of the indigenous people of the study area.

\section{Acknowledgement}

The authors wish to acknowledge the fund provided by the TETFund through the Ekiti State University, Ado-Ekiti, Nigeria, for this study.

\section{References}

Abubakar, S., Ogbadu, G. H., Usman, A. B. Segun, O. Olorode, O. \& Samirah, I. U. (2012). "The underutilized vegetable plants of the federal capital territory (FCT) Abuja of Nigeria". International Journal of Development and Sustainability, 1(3): 634-643. Adebooye, O.L. \& Opabode, J.T. (2004). "Status of conservation of the indigenous leaf vegetables and fruits of Africa". African Journal of Biotechnology. 3(12): 700-705.

Adedokun, M. O. and Kayode, G. M. (2019).” Local Leadership Styles and Community Development: A Study of the Irasa Community of Ado-Ekiti, Nigeria". Budapest International Research and Critics in Linguistics and Education Journal 2(4), 27 31. DOI : https://doi.org/10.33258/birle.v2i4.469

Ajiboye, A. A., Fadimu, O. I., Ajiboye, M. D., Agboola, D. A., Adelaja, A. B. \& Bern, A. A. (2014). "Phytochemical and nutritional constituents of some commonvegetables in south west Nigeria". Global Journal of Sc. Fron. Res. XIV (III) version 1.

Ali, M.S. Jamous, R.M. Al-Oshafie, J.H. Elgharaba, W.A. Kherfan, F.A. Qarariah, K.H. Khdair, I.S. Soos, I.M. Museh, A.A. Isa, B.A. Herzallah, H.M. Khlaif, R.B. Aiash, S.M. Swaiti, G.M. Abuzahra, M.A. Haj-ali, M.M. Saifi, N.A. Azem, H.K. \& Nasrallah, H.A. (2008). "Traditional Knowledge of wild edible plants used in Palestine. (Northern West Bawl): A Comparative study". Journal of Ethnobiology and Ethnomedicine. 4(13): 5-11.

Arowosegbe, S. Olanipekun, M.K. \& Adeloye, I.A. (2018). "Ethnobotanical Survey of Indigenous leafy vegetables consumed in Ekiti State, Nigeria". European Journal of Biology and Medical Science Research. 6(1):7-14.

Ayeni, M. J., Kayode, J. \& Oloruntobi, T. O. (2018). "An interactive survey on the efficacy of medicinal plants in the management of malaria in Kaduna metropolis of Nigeria". Nigerian Journal of Botany 31(1), 17-31.

Bonger, F. \& Popma, J. M. (1998). "Ethnobotanical research and traditional health in developing countries, Plants, People and Culture". W.H. Freeman and Co; Conservation Resources by Plant- Talk Ltd. UK Plant - Talk Periodical.

Bvenura, C. \& Afolayan, A.J. (2014). "Ethnobotanical survey of wild vegetables in Mbashe and Nkonkobe municipalities, Eastern Cape Province, South Africa". Actta Botanical 
Gallica. 161:189-199.

emails: birex.journal@gmail.com

Campton, R. (2008). "The economic value of wildlife in eastern and central Africa".

Research Paper Volume 23, Institute of Development Studies, University of Dares Salaam, Tanzania.

Chadha, M.L. and Oluoch, M. O. (2002). "Home based vegetable gardens and other strategies to overcome micronutrient malnutrition in developing countries". Food, Nutrition and Agriculture 32: 7-13.

Coolborn, A.F. \& Adegbemisipo, A.A. (2018). "Medicinal Vegetal use by traditional healers in Ekiti State of Nigeria for diabetes treatment". International Journal of Pharmacy Research and Technology. 8(10):21-28.

Harper, J. L. (1977). "Population Biology of Plants". Academic Press, London, 892pp.

Kalemba. P. (2007). "Wild plants and Tradition in East Africa". Unpublished manuscript, Department of Botany, University of Kinshasa, DRC.

Kayode, J. (1999). "Phytosociological investigation of compositae weeds in abandoned farmlands in Ekiti State, Nigeria". Compositae Newsletter 34:62-68.

Kassim, S. (2009). "Wildlife and Food Security". Paper prepared for the Forestry Department, Food and Agriculture Organization, Rome, Italy.

Kayode, J. (2005). "Checklist of roadsides compositae weed species in Southwestern Nigeria". Compositae Newsl. 42: 109-114.

Kayode, J. Omotoyinbo, M. A., Ayeni, M. J. \& Oyedeji, A. A. (2015). "Stem Barks and Roots Extravitism in Ekiti State Nigeria: Need for Conservation as a Sustainable Innovation in Healthcare Management in Rural Areas". American Journal of BioScience 3 (2), 28-33. doi: 10.11648/j.ajbio.20150302.11

Kayode, J. Odesola, A.F. Ayeni, M. J. \& Awoyemi, S.B. (2016). "Survey of botanicals used as pesticides by the rural farmers of Ekiti State, Nigeria". International Journal of Biological Papers. 1: 12-17.

Kebede, A. Tesfaye, W. Fentie, M.\& Zewide, H. (2017). “An Ethnobotanical Survey of wild edible plants commercialized in Kefira market, Dire Dawa city, Eastern Ethiopia". Plant, 5 (2): 42-46. doi: 10.11648/j.plant.20170502.13

KDAH (2015). "Tips to prevent lifestyle diseases". Kokilaben Dhirubhai Ambani Hospital. https://www.kokilabenhospital.com/blog/tips-to-prevent-lifestyle-diseases/

Labadarios, D., Steyn, N., Maunder, E., Gericke, G., Swart, R., Huskinson, J., Dannhhauser, A., Voster, H. H., Nesamvuni, A. E. \& Nel, J. H. (2005). "The National Food consumption Survey (NFCS), South Africa”, Public Health Nutrition. 8(5): 533-543.

Lwoga, E.T. Ngulube, P. Stilwell, C. (2010). "Managing indigenous knowledge for sustainable agricultural development in developing countries knowledge management approaches in the social context". The International Information and Library Review. 42: 174-185.

Maunder, E.M. \& Meaker, J. L. (2007). "The current and potential contribution of homegrown vegetables to diets in South Africa". Water SA. 33(3): 401-406.

Modi, M., Modi, A. T. \& Hendriks, S. (2006). "Potential role of wild vegetables in household food security : A preliminary case study in Kwazulu-Natal, South Africa”. African Journal of Food Agriculture Nutrition and Development. 6(1): 1-13.

Neudeck, L. Avelino, L. Bareetseng, P. Ngwenya, B.N. Teketay, D. \& Motsholapheko, M.R. (2012). "The conservation of edible wild plants to food security, Dietary diversity and income of households in Shorobe village, Northern Botsawana". Ethnobotany Research and Applications. 10: 449-462.

Nnamani, C.V. Oselebe, H.O. \& Okporie, E.O.( 2017). “Aspect of ethnobotany of traditional leafy vegetables utilized as human food in rural tropical communities". Animal Resources International. 7: 1110-1115. 
NPC, 2010. "Population and Housing Census, Priority Table Volume IV, Population Distribution by Age and Sex". National Population Commission (NPC), Abuja, Nigeria.

Obembe, M. O. and Kayode, J. (2019). "Evaluation of the Insecticidal Properties of Cassia alata L. Against Cowpea Weevil, Callobruchus maculatus Fab. (Coleoptera: Bruchidae)". Budapest International Journal in Exact Sciences 1 (4), 84-92. DOI : https://doi.org/10.33258/birex.v1i4.481

Oberbaner, S. F. \& Strain, B. R. (1985). "Effects of light regimeon growth and physiology of P. microloba (Mimosaceae) in Costa Rica". Journal of Tropical Ecology 1, 303-320.

Ogunrotimi, D. G., Kayode, J. \& Odesola, F. A. (2018). "Ethnobotany and conservation of indigenous vegetables in Ekiti State, Nigeria". Singapore Journal of Scientific Research. 8(1): 8-13.

Ojelel, S. \& Kakudidi, E.K. (2015). "Wild edible plant species utilized by a subsistence farming community in Obalanga Sub-County, Amuria District, Uganda". Journal of Ethnobiology and Ethnomedicine. 11(7): 1-8.

Quora (2019). "What is the largest family of plants?" https://www.quora.com/What-is-the-largest-family-of-plants

Saqib, Z. Malik, R.N. Shinwari, M.I. \& Shinwari, Z. (2011). "Species richness, ethnobotanical species richness and human settlements along a Himalayan altitudinal gradient: Prioritizing plant conservation in Palas valley, Pakistan”. Pakistan Journal of Botany. 43: 129-133.

Vaishali, S.K. \& Jadhvah, V.D. (2013).’Traditional leafy vegetables: A Future Herbal Medicine". International Journal of Agricultural and Food Science. 3(2): 56-58.

Van der walt, A.M. Mossanda, K.S. Jwan, S.D. Swart, W.J. \& Bezuidenhout, C.C. (2005). "Indigenous African Food Plants: Vehicles of diseases or sources of protection? ". Indilinga: Africa Journal of Indigenous Knowledge Systems. 4(1): 270-279. 